\title{
In-Situ Measurements of Single Walled Carbon Nanotube Growth Reveal the Structures of Active and Inactive Catalyst Nanoparticles
}

\author{
$\underline{\text { Hsin-Yun Chao }}^{1,3}$, Hua Jiang $^{2}$, John Cumings ${ }^{1}$ and Renu Sharma ${ }^{3}$
}

1. Materials Science and Engineering, University of Maryland, College Park, MD, United States.

2. Department of Applied Physics, Aalto University School of Science, P.O. Box 15100, FI-00076 Aalto, Finland

3. Physical Measurement Laboratory, National Institute of Standards and Technology, Gaithersburg, MD, United States.

Single walled carbon nanotubes (SWNT) remain a promising material for enhancing performance of devices. Understanding the growth mechanisms are of vital importance to ensure structure control of SWNT. Recently, it was shown that phase change occurs in cobalt catalysts during SWNT growth which fluctuates between cobalt and carbide phases [1]. Furthermore, previous work supported by density functional theory (DFT) calculations suggest that in order for SWNT growth to occur, the catalyst transitions to the carbide $\mathrm{Co}_{2} \mathrm{C}$ phase with a Co terminated $\{020\}$ surface that allows graphene anchoring and a Co-C terminated $\{210\}$ surface that enables liftoff to occur for the formation of the nanotube [2]. However, empirical studies have yet to identify the structures of active, inactive and deactivated catalyst nanoparticles at the atomic scale.

In this study, we performed statistical analysis focusing on a number of cobalt catalyst particles with atomically resolved dynamic imaging using an in-situ environmental transmission electron microscope (ETEM) to determine their phase and corresponding zone axes. In addition, we distinguished between the active, inactive and deactivated particles by direct observation to determine factors affecting growth.

SWNT growth was performed with Co catalyst and $\mathrm{MgO}$ support on $\mathrm{SiO}_{2}$ membrane chip compatible with a MEMS heating holder (DENS Solutions). Pressures of $0.02 \mathrm{~Pa}$ to $0.005 \mathrm{~Pa}$ of $\mathrm{C}_{2} \mathrm{H}_{2}$ were used as the carbon source at $700{ }^{\circ} \mathrm{C}$ to $900{ }^{\circ} \mathrm{C}$ in the ETEM, equipped with a monochromated FEG and an image corrector, operated at $80 \mathrm{KeV}$. A charge coupled device (CCD) was used to record HRTEM images and videos. The $d$-spacings and angles were measured from the fast Fourier transform (FFT) of atomic resolution images of the target catalyst nanoparticles extracted from the videos. A custom algorithm, Crystalball, was used for unambiguous phase determination [3].

As previously reported, catalyst phases can undergo many transitions that result in varied growth rates [1]. In Fig. 1a, initially we observed a catalyst in the orthorhombic $\mathrm{Co}_{3} \mathrm{C}$ phase with $(002),(\overline{2} 0 \overline{1})$ and (201) planes, which are favorable planes for SWNT growth. A cap of SWNT formed around the catalyst and propagation initiated. After 5 seconds, the active catalysts transitioned into a deactivated catalyst that terminated the SWNT growth with the same carbide phase but reoriented into planes of $(022),(\overline{2} 11)$ and $(\overline{2} \overline{1} \overline{1})$ and the zone axes changed from [010] to [0 $\overline{1} 1]$. This example supports that the propagation of SWCNT can be affected by reorientation of catalyst nanoparticles with varied planes and zone axes.

Additionally, it is possible for a carbide-dominant catalyst to transition into a cobalt oxide-dominant phase. Fig. 1b shows a previously active catalyst nanoparticle for SWNT growth. During the in-situ observation period, the SWNT growth was attenuated. The catalyst retained the orthorhombic $\mathrm{Co}_{2} \mathrm{C}$ phase, but with non-favorable growth planes (101) and (101) on the surface. After a period of 10 
seconds, the catalyst transitioned into a cubic $\mathrm{Co}_{3} \mathrm{O}_{4}$ phase with planes of (220), (11 $\left.\overline{3}\right)$, and ( $(\overline{1} \overline{1} \overline{3})$. Thus, the transition to an oxide phase appears to result in the deactivation of the catalyst.

Our results show that active growth is only favorable when the catalyst is in the carbide phase.

Moreover, the $\{020\}$ anchoring surface coupled with the $\{210\}$ liftoff surface increased the success rate of propagation which was predicted by theoretical calculations reported earlier [1]. For cases where a catalyst was observed with the cobalt oxide phase, the catalyst was either inactive from the start with no formation of SWNT or became deactivated after tube growth and exhibited no further elongation (Fig 2a). Instances where potentially active planes on the catalyst had no visible tube growth, the active planes were obscured by amorphous carbon (Fig. 2b). All active catalysts observed were oriented along either the $\langle 100\rangle$ or $\langle 110\rangle$ zone axes, whereas the inactive catalysts had a wider range of zone axes. Additionally, inactive sites consisted of oxidized cobalt rather than pure cobalt.

To summarize, the in-situ ETEM method enables us to observe dynamic evolutions of catalysts in real time. We were able to obtain a sizable dataset for statistical analysis with respect to the types of catalyst structures that promote or inhibit growth. It is shown that carbide-dominant catalysts with favorable adhesion energy for graphene, i.e. $\{020\}$ and $\{210\}$ offer a consistent condition for growth unless obstructed by amorphous carbon. For oxide-dominant catalysts, the growth becomes absent or stopped. Ultimately, we hope these findings will aid in designing catalysts with higher yield for the future production of SWNT. Comprehensive results from statistical nanoparticle analysis will be presented.

\section{References:}

[1] Z. Hussaini et al, Ultramicroscopy 186 (2018), p. 139-145.

[2] M. Picher et al, Nano Letters 14 (2014), p. 6104-6108.

[3] Crystalball Plus - 2011, https://notabug.org/stefano-m/crystalballplus (accessed Feb. 12, 2019).

a)

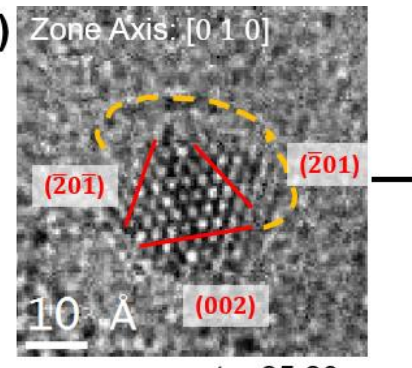

$\mathrm{t}=25.20 \mathrm{~s}$

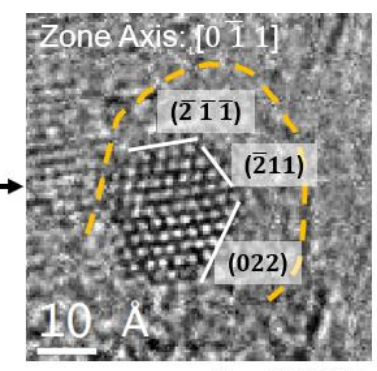

$\mathrm{t}=29.87 \mathrm{~s}$ b)

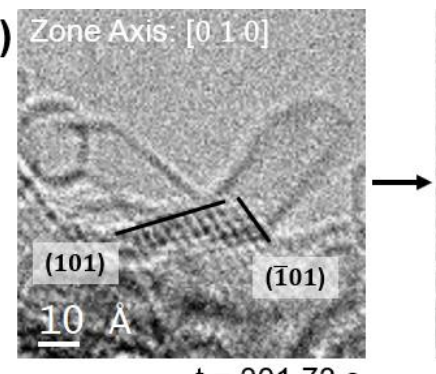

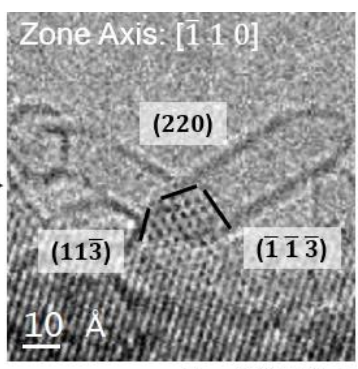

$\mathrm{t}=311.86 \mathrm{~s}$

Figure 1. Stages of growth deactivation include: a) reorienting from favorable to non-favorable growth planes of $\mathrm{Co}_{3} \mathrm{C}$ and $\mathbf{b}$ ) transitioning from $\mathrm{Co}_{2} \mathrm{C}$ carbide to $\mathrm{Co}_{3} \mathrm{O}_{4}$ oxide phase. Red planes indicate those favorable for SWNT growth and yellow dashed lines outline the SWNT profile.

a)

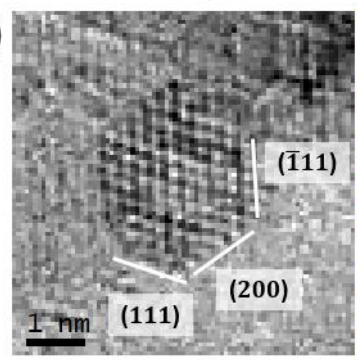

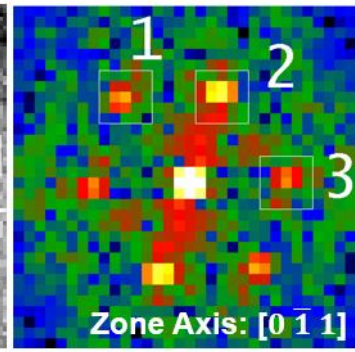

b)

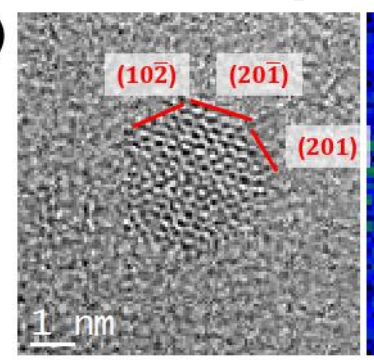

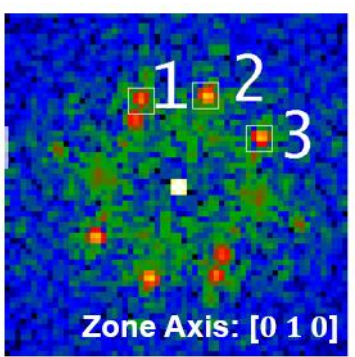

Figure 2. Inactive catalyst nanoparticles include: a) $\mathrm{CoO}$ phase with non-favorable planes and b) $\mathrm{Co}_{3} \mathrm{C}$ phase with favorable planes but embedded in amorphous carbon. Planes are labeled with corresponding FFT and zone axes. 